\title{
On the theory of transport phenomena in ferrofluids. Effect of chain-like aggregates
}

\author{
A.Yu. Zubarev* \\ Department of Mathematical Physics, Urals Federal University, Lenin Ave, 51, 620000, Ekaterinburg, Russia
}

\section{A R T I C L E I N F O}

\section{Article history:}

Received 9 February 2012

Received in revised form 23 May 2012

Available online 23 August 2012

\section{Keywords:}

Ferrofluids

Chains

Transport phenomena

\begin{abstract}
A B S T R A C T
The paper deals with the theoretical study of the effect of chain-like aggregates on diffusion and magnetophoretic transport in ferrofluids. Analysis shows that the appearance of the chains leads to a strong anisotropy of the diffusion transport - the coefficient of diffusion in the direction of applied magnetic field is significantly more than that in the perpendicular direction. The presence of the chains in a ferrofluid strongly affects the coefficient of the particle magnetophoresis.
\end{abstract}

(C) 2012 Elsevier B.V. All rights reserved.

\section{Introduction}

Ferrofluids are colloidal suspensions of single-domain ferromagnetic particles in a carrier liquid. The typical size of the particles in modern ferrofluids is about $10-20 \mathrm{~nm}$. These systems are attracting considerable interest from researchers and engineers due to a rich set of unique physical properties, which find active applications in various modern industrial, biological and medical technologies. Diffusion and magnetophoretic phenomena in ferrofluids determine many important properties of these systems and their behavior in various technological applications. That is why a detailed study of transport phenomena in ferrofluids is important both from the point of view of the fundamental theory of magnetic fluids and from the viewpoint of the development of a scientific background for their practical usage.

In the case of very dilute systems, where any interactions between particles are negligible, the coefficient of the ferroparticle diffusion can be determined by the classical Einstein formula for the diffusion coefficient of a Brownian particle. When the interparticle interactions are significant, this coefficient depends on the particle concentration and applied magnetic field as well. Theoretical investigations of the diffusion and magnetophoretic transport in magnetic fluids with an account of magnetic, hydrodynamic and sterical interactions between particles are presented in Refs. [1-4]. It is shown that in a magnetic field the diffusion coefficient is anisotropic-its magnitude along the field differs from that in the perpendicular direction.

The models [1-4] deal with the case of moderate and weak interparticle interactions, when the particles cannot form any heterogeneous structures. However observations demonstrate that linear chains, bulk dense "drops" and other internal structures occur in many modern ferrofluids. An applied magnetic field stimulates the appearance of these structural transformations (see, for example, Refs. [5-9]).

The typical size of the bulk drops in ferrofluids is about several microns. Usually they are clearly seen in optical microscopes [5-7]. Since the wavelength of visible light is much more than the size of the particles in ferrofluids, the linear chains can not be detected optically, but they have been observed with the help of electron microscopes [8,9].

\footnotetext{
* Tel.: +7 3433507541

E-mail address: andrey.zubarev@usu.ru.
} 
The appearance of the internal heterogeneous structures significantly affects the magnetic, rheological, optical and other properties of ferrofluids $[10,11]$. One can expect an influence of these structures on the transport properties and phenomena in these systems.

Many modern ferrofluids are polydisperse, often with a wide distribution over the particle size [10]. Particles of mean size in them are too small to form any heterogeneous aggregates and structures. Usually the chain-like and drop-like aggregates consist of the largest particles, whose concentration in ferrofluids, as a rule, is small. That is why the effect of the largest particles and heterogeneous structures on the transport phenomena in traditional ferrofluids is rather weak.

However, various ferrofluids, consisting of strongly interacting particles, which are able to form chains and other structures, have been synthesized for the past decade. Ferrofluids consisting of cobalt [11] as well as of cluster particles $[12,13]$ are examples of fluids with a strong magnetic interparticle interaction.

In these and similar ferrofluids, a significant fraction of the particles can be united into chains or other heterogeneous aggregates. That is why the effect of the aggregates on the transport phenomena in these systems can be very strong. Analysis of the magnetodiffusion and magnetophoretic transport in ferrofluids with chains is important also in connection with the problem of magnetic separation of polydisperse systems with the aim to remove the largest particles, which can form heterogeneous aggregates.

The goal of this work is the analysis of the effect of the chain-like aggregates on the diffusion and magnetophoretic (i.e. under the action of spatially inhomogeneous magnetic field) transport in ferrofluids with strongly interacting particles.

\section{Physical model and the main approximations}

We will use a simple model of the chain-like aggregates, developed in Ref. [14]. In spite of simplifications, this model has allowed us to describe rheological properties of various magnetic fluids with the single-domain and cluster particles as well [15-18].

In the framework of the model [14] the ferrofluid particles are considered as identical ferromagnetic spheres with diameter $d$ and magnetic moment $m$, fixed in the particle body. It is supposed that the particles can form only linear chainlike structures. Any interactions between the chains are ignored.

Following Refs. [14-18] we will suppose that the energy of the dipole-dipole interaction between the nearest particles in the chain is significantly more than the thermal energy $k T$. This condition is necessary for the appearance of any heterogeneous aggregates in a ferrofluid. We neglect the thermal fluctuations of the chain shape and orientations of the particle moments in the chain. In other words, the chain is modeled by a straight rigid rod-like aggregate; the particle magnetic moments are aligned along the aggregate axis. The criterion for applicability of this approximation is discussed in Ref. [14]. In part, it is shown that this approximation is justified when the inequalities $\varepsilon>\kappa, \varepsilon \gg 1$ are fulfilled. Here $\varepsilon=\frac{\mu_{0}}{2 \pi} \frac{m^{2}}{d^{3} k T}, \kappa=\mu_{0} \frac{m H}{k T}, H$ is the local magnetic field in the sample, $\mu_{0}$ is the vacuum magnetic permeability. The first inequality means that the energy of magnetic interaction between the particles is more than the energy of their interaction with the magnetic field.

Next, we will take into account interaction only between the nearest particles in the chain.

Approximation of the rigid rod-like chains, of course, is a very strong simplification. A model of the flexible chains in an arbitrary magnetic field has been developed in Ref. [19]. However, this model leads to complicated and cumbersome calculations. At the same time results [19] show that the model of the rod-like aggregates allows one to get quite reasonable estimates for ferrofluid equilibrium magnetization. It has been noted already, that this model allows a description of rheological phenomena in various ferrofluids. It gives us a background to consider this model as a robust foundation for the description of the transport phenomena in ferrofluids.

\section{Thermodynamical functions of magnetic fluid}

Let us consider a unit representative volume of the ferrofluid. We will suppose that this volume contains a tremendous number of the particles; its size is much less than the characteristic distance of change of the particle concentration. As is well known, introduction of the small representative volume is necessary for the usage of continuous methods of description of transport and other nonequilibrium phenomena.

We will denote the number of $n$-particle chains in this volume as $g_{n}$. Let us suppose that at each moment of time the thermodynamical state of every elementary volume of the fluid can be considered as in equilibrium. In the framework of the chosen approximations, the free energy $F$ of the unit volume of the fluid can be presented as [14]:

$$
F=k T \sum_{n=1}^{\infty}\left[g_{n} \ln \frac{g_{n} v}{\mathrm{e}}-g_{n} \varepsilon(n-1)-g_{n} \ln \frac{\sinh (\kappa n)}{\kappa n}\right] .
$$

The first term in the square brackets (1) corresponds to the entropy of an ideal gas of the non-interacting chains. The second term describes the energy of interaction of the neighbor particles in the chains. The third term presents the Langevin free energy of the chain interaction with the local magnetic field $H$. 
The equilibrium state of the elementary unit volume corresponds to the distribution function $g_{n}$ providing the minimum of the free energy $F$ under the normalization condition

$$
\sum_{n=1}^{\infty} n g_{n}=\frac{\varphi}{v} .
$$

Here $\varphi$ is the volume concentration of the particles in the unit elementary volume, $v$ is the particle volume.

Standard minimization of $F$ (see details in Ref. [14]) leads to the following expression for the function $g_{n}$ :

$$
g_{n}=\frac{x^{n}}{v} \frac{\sinh (\kappa n)}{\kappa n} \exp (-\varepsilon) .
$$

Here $x$ is an undetermined Lagrange multiplier. It can be calculated by substituting (3) into condition (2). As a result we get [14]:

$$
\begin{aligned}
& x=\frac{2 y \cosh (\kappa)+\sinh (\kappa)-\sqrt{(2 y \cosh (\kappa)+\sinh (\kappa))^{2}-4 y^{2}}}{2 y}, \\
& y=\kappa \varphi \exp (\varepsilon) .
\end{aligned}
$$

Combining Eqs. (3), (4) and (1), one can get an explicit form for the free energy $F$ as a function of the local concentration $\varphi$ and the local magnetic field $H$. By using this form, we can calculate any thermodynamical functions of the magnetic fluid. For example, the local magnetization $\mathbf{M}$ has the following form:

$$
\begin{aligned}
& \mathbf{M}=-\frac{\mathbf{H}}{\mu_{0} H} \frac{\partial F}{\partial H}=m \sum_{n=1}^{\infty} n L(\kappa n) g_{n} \frac{\mathbf{H}}{H}=\chi \mathbf{H} \\
& \chi=12 \frac{\varepsilon}{\kappa} v \sum_{n=1}^{\infty} n L(\kappa n) g_{n} .
\end{aligned}
$$

Here $\chi$ is the magnetic susceptibility of the ferrofluid. After simple calculations we come to the following expression for the chemical potential of the particles:

$$
\mu=v \frac{\partial F}{\partial \varphi}=k T(\ln x-\varepsilon) .
$$

\section{Flux of the particles}

According to the general Einstein-Batchelor formula [20], the density of the flux $\mathbf{j}_{n}$ of the particles united in the $n$-particle chains can be presented as follows:

$$
\mathbf{j}_{n}=-n g_{n} \boldsymbol{\beta}_{n}: \nabla \mu \text {. }
$$

Here $\beta_{n}$ is the tensor of the hydrodynamical mobility of the chain. The explicit form of components of $\beta_{n}$ will be discussed below. The tensor character of the mobility is explained by the anisotropic shape of the chain.

The chemical potential $\mu$ of the particles depends on the particle volume concentration $\varphi$ and on the absolute value $H$ of the local magnetic field. Thus, one can present:

$$
\nabla \mu=\frac{\partial \mu}{\partial \varphi} \nabla \varphi+\frac{\partial \mu}{\partial H} \nabla H .
$$

The relation (8) must be augmented by the Maxwell equations for the magnetic field $\mathbf{H}$. These equations can be written down as

$$
\operatorname{div}[\mathbf{H}(1+\chi)]=0, \quad \operatorname{rot} \mathbf{H}=0 .
$$

The first equation of (9) can be presented in the following form:

$$
(1+\chi) \operatorname{div} \mathbf{H}+\frac{\partial \chi}{\partial \varphi}(\mathbf{H} \cdot \nabla) \varphi+\frac{\partial \chi}{\partial H}(\mathbf{H} \cdot \nabla) H=0
$$

Combining Eqs. (7)-(10), one can find an expression for the flux $\mathbf{j}_{n}$ through components of the vector $\nabla \varphi$. The total flux of the particles is:

$$
\mathbf{j}=\sum_{n=1}^{\infty} \mathbf{j}_{n} .
$$

Generally speaking, Eq. (10) is non-linear and does not have an analytical solution. Below we consider two simple, however quite typical, situations when this solution can be obtained. 


\subsection{Magnetic field is parallel to the gradient of concentration}

Let us denote the mean magnitudes of the magnetic field and the particle concentration in some physically small volume as $H_{0}$ and $\varphi_{0}$ respectively. In this volume the field and concentration can be presented as

$$
H=H_{0}+h, \quad \varphi=\varphi_{0}+\varphi^{\prime} .
$$

We will suppose that the following strong equations $|h| \ll H_{0},\left|\varphi^{\prime}\right| \ll \varphi_{0}$ are fulfilled. Indeed, under the condition that the field and concentration are spatially continuous functions, we can always choose the above-mentioned volume small enough to provide small deviations of $H$ and $\varphi$ from $H_{0}$ and $\varphi_{0}$ inside this volume. Since the relations (8)-(10) are spatially local and relate to each physical point (i.e. to each small volume) of the fluid, usage of the last inequalities in this volume cannot lead to incorrect results.

Let us introduce a Cartesian coordinate system with the axis $O x$, aligned along the local gradient of concentration $\nabla \varphi$. We suppose that the locally mean field $\mathbf{H}_{0}$ is also aligned along this axis. The symmetry consideration shows that the perturbation $\mathbf{h}$ to $\mathbf{H}_{0}$ also has only a component along $O x$.

Taking it into account, after simple transformations in the linear approximation in $h$ and $\varphi^{\prime}$, one can get from (10) that

$$
\begin{aligned}
& \frac{\partial h}{\partial x}=-B \frac{\partial \varphi^{\prime}}{\partial x}, \\
& B(\varphi, H)=H \frac{\partial \chi}{\partial \varphi} \frac{1}{1+\chi+H \frac{\partial \chi}{\partial H}} .
\end{aligned}
$$

The magnitude of the susceptibility $\chi$ and its derivatives are determined here for the locally mean magnitudes $H_{0}$ and $\varphi_{0}$.

Combining relations (13) with (7), (8) and (11), we get

$$
\begin{aligned}
& j=-\frac{D_{\|}}{v} \frac{\partial \varphi}{\partial x} \\
& D_{\|}=\sum_{n=1}^{\infty} v n g_{n} \beta_{n \|}\left(\frac{\partial \mu}{\partial \varphi}-B \frac{\partial \mu}{\partial H}\right) .
\end{aligned}
$$

Here $\beta_{\mathbf{n} \|}$ is the coefficient of the hydrodynamical mobility of the $n$-particle chain along its axis, $D_{\|}$is the effective coefficient of diffusion, corresponding to the parallel orientation of the magnetic field $\mathbf{H}$ and gradient of the concentration $\varphi$. Because of the above-mentioned reasons we make no distinction here between $\varphi$ and $\varphi_{0}$, and $H$ and $H_{0}$.

\subsection{The field is perpendicular to the gradient of concentration}

After the same considerations we get $\frac{\partial h}{\partial x}=0$. The relations (7) and (11) now give

$$
\begin{aligned}
& j=-\frac{D_{\perp}}{v} \frac{\partial \varphi}{\partial x}, \\
& D_{\perp}=\sum_{n=1}^{\infty} v n g_{n} \beta_{n \perp} \frac{\partial \mu}{\partial \varphi} .
\end{aligned}
$$

Here $\beta_{n \perp}$ is the coefficient of the chain mobility in the direction perpendicular to its axis.

Let us discuss now components of the tensor $\boldsymbol{\beta}_{n}$ of the chain mobility. The mobility $\beta_{1}$ of the single particle is determined by the classical Stokes formula. Because of the complicated shape of the chain, a strict calculation of the chain mobility is impossible. Here, in order to get physically reasonable estimates, we will model the chain by an ellipsoid of revolution with the major and minor axes equal to $2 n a$ and $a$ respectively. Here $a$ is the hydrodynamical diameter of the particle, determined with the surface shell on the particle. It should be noted that the ellipsoidal model has been used in Refs. [14-18] for calculations of rheological characteristics of ferrofluids.

The expressions for components of the coefficient of mobility of an ellipsoid of revolution can be found, for example, in overview [21]. For the longitudinal and perpendicular directions, with respect to the ellipsoid axis, these components have the following forms

$$
\begin{array}{ll}
\beta_{n \|}=\frac{1}{16 \pi \eta}\left(\gamma+\alpha_{1} n^{2} a^{2}\right), & \beta_{n \perp}=\frac{1}{16 \pi \eta}\left(\gamma+\alpha_{2} a^{2}\right) \\
\alpha_{1}=\int_{0}^{\infty} \frac{\mathrm{d} \lambda}{\left(n^{2} a^{2}+\lambda\right) \Delta(\lambda)} & \alpha_{2}=\int_{0}^{\infty} \frac{\mathrm{d} \lambda}{\left(a^{2}+\lambda\right) \Delta(\lambda)} \\
\gamma=\int_{0}^{\infty} \frac{\mathrm{d} \lambda}{\Delta(\lambda)}, & \Delta(\lambda)=\sqrt{\left(n^{2} a^{2}+\lambda\right)}\left(a^{2}+\lambda\right) .
\end{array}
$$

Here $\eta$ is viscosity of the carrier liquid. 

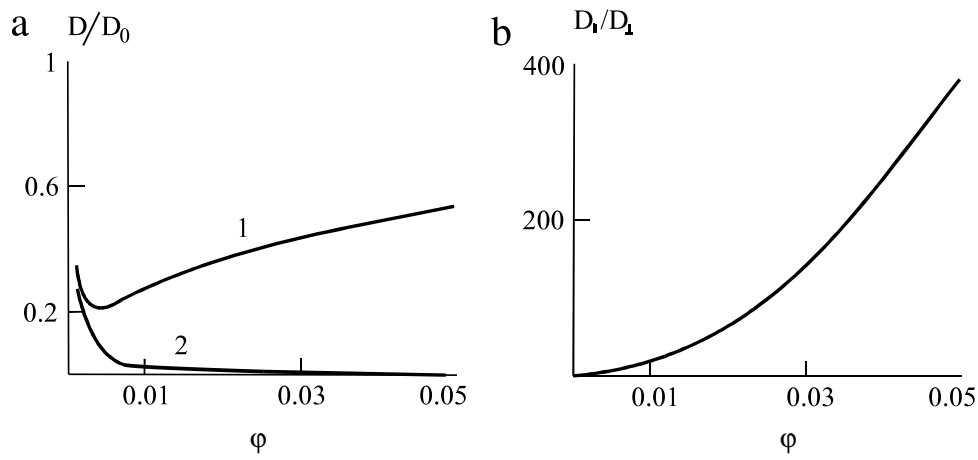

Fig. 1. (a) The dimensionless coefficients of longitudinal $D_{\|}$(line 1 ) and perpendicular $D_{\perp}$ (line 2) coefficients of diffusion vs. the particle volume concentration $\varphi ; D_{0}$ is the coefficient of diffusion of a single particle, calculated using the Einstein formula. (b) Ratio of these coefficients. Dimensionless magnitudes of the field and energy of magnetic interaction between particles $\kappa=2$ and $\varepsilon=6$ respectively.
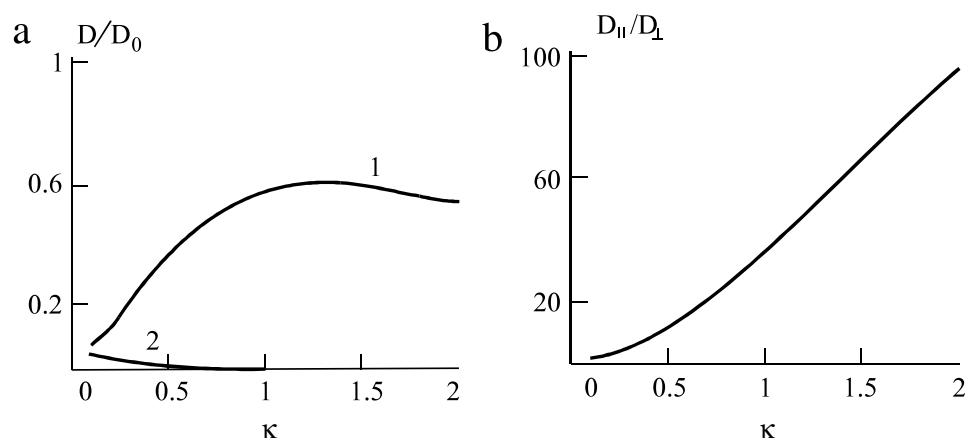

Fig. 2. (a) The dimensionless diffusion coefficients vs. dimensionless magnetic field $\kappa$. Notations are the same as in Fig. 1. (b) The ratio of the diffusion coefficients as a function of $\kappa$. Volume concentration of the particles and parameter of their magnetic interaction $\varphi=0.025$ and $\varepsilon=6$ respectively.

The results of calculations of the coefficients $D_{\|}$and $D_{\perp}$ as functions of the concentration $\varphi$ are presented in Fig. 1 .

The dependence of $D_{\perp}$ on concentration $\varphi$ is explained by a combination of two factors. First, the magnetic attraction between particles and their aggregation into the chains decreases the particle chemical potential $\mu$ and the derivative $\frac{\partial \mu}{\partial \varphi}$ as compared with the case of single particles. Second, the appearance of the chains reduces the effective hydrodynamical mobility of the particles in the direction perpendicular to the field $\mathbf{H}$. Both of these factors lead to the decreasing dependence of $D_{\perp}$ on $\varphi$. The longitudinal coefficient $D_{\|}$, besides these factors, is determined by attraction of the particles to the region with relatively high magnitude of the local field $H$, i.e. to the regions where the concentration $\varphi$ is relatively small. Obviously, this factor increases the effective diffusion of ferroparticles. Combinations of all these mechanisms lead to a non-monotonic dependence of $D_{\|}$on $\varphi$.

Fig. 2 demonstrates the dependence of both of these diffusion coefficients on the dimensionless magnetic field.

The monotonic decrease of $D_{\perp}$ with the field is explained by an increase of the particle attraction and the appearance of longer chains under the field action. These factors lead to a decrease of the particle chemical potential and their effective hydrodynamical mobility. Both of these factors decrease the diffusion coefficient.

The coefficient $D_{\|}$of the longitudinal diffusion is determined, besides these factors, by attraction of the particles to regions where the local field is high, i.e. the local concentration is small. The combination of these factors leads to a non-monotonic dependence of $D_{\|}$on the field.

Figs. 1 and 2 demonstrate strong anisotropy of the diffusion transport. In the considered regions of $\varphi$ and $\kappa$, the ratio of the coefficients $D_{\|}$and $D_{\perp}$ reaches one to two orders of magnitude. The microscopic nature of this strong difference between $D_{\|}$and $D_{\perp}$ lies, first, in the difference of hydrodynamical mobilities of the chains in the directions along and perpendicular to the chain axis; second, in the attraction of the particles to the region with relatively high magnetic field, i.e. to the regions where the particle concentration is relatively small. The last mechanism of the diffusion anisotropy takes place also for ferrofluids with individual particles; however for the fluids with chains its effect is much stronger. Enhancement of this effect due to the appearance of the chains is explained by the high correlation of the particle magnetic moments in a chain. As a result, the moments of the particles in the chains are more strongly orientated by the field than the moments of the single particles. Therefore, the mean energy of interaction of the aggregated particles with the field is stronger than that for the individual particles. 

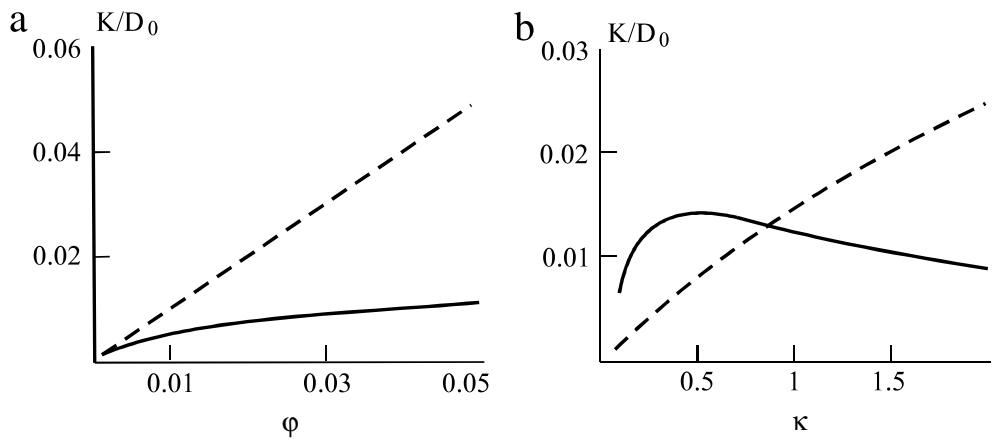

Fig. 3. Dimensionless coefficient of magnetophoresis in the direction perpendicular to the applied magnetic field vs (a) the particle volume concentration, when $\kappa=2$; (b) applied magnetic field, when $\varphi=0.025$. Dimensionless parameter of the dipole-dipole interaction between particles $\varepsilon=6$. Solid lines-calculations according to (16) taking into account presence of the chains; dashed ones-model (17) of individual non-interacting particles.

\section{Magnetophoresis}

Let us consider now a model situation, assuming that the concentration $\varphi$ of the particles is homogeneous, and their transport takes place due to spatial inhomogeneity of the applied magnetic field $\mathbf{H}$. We will consider the situation when the gradient $\nabla H$ of the absolute value of the field is perpendicular to $\mathbf{H}$. In this case the magnetophoretic transport takes place in the direction perpendicular to the orientation of the chain axes.

Combining relations (7), (8) and (11), we now get

$$
\begin{aligned}
& j=K \nabla \kappa, \\
& K=-\sum_{n=1}^{\infty} n g_{n} \beta_{n \perp} \frac{\partial \mu}{\partial \kappa} .
\end{aligned}
$$

Here $K$ can be considered as the coefficient of the particle magnetophoresis. Since the chemical potential $\mu$ decreases with the dimensionless field $\kappa$, the coefficient $K$ is positive.

In the approximation of the individual non-interacting particles, one should put in relation (1) $g_{1}=\varphi / v$ and $g_{n}=0$ when $n \neq 1$. In this case

$$
K_{0}=\frac{\varphi}{v} \beta_{0} L(\kappa), \quad \beta_{0}=6 \pi \eta a .
$$

Here index 0 denotes the coefficients of magnetophoresis and hydrodynamical mobility of individual non-interacting particles.

Calculations of parameters $K$ are presented in Fig. 3. When the dimensionless field $\kappa$ is small enough, the appearance of the chains increases the magnetophoretic coefficient. This effect is explained by an increase, due to correlations of the particle moments in the chains, of the mean magnetic moment of the particles and, therefore, enhancement of their interaction with the field. However, an increase of the field induces an increase of the characteristic size of the chains and, therefore, a decrease of their hydrodynamical mobility. When $\kappa$ is large enough, the last factor dominates and the coefficient $K$ decreases with the field.

\section{Conclusion}

In the framework of the simple approximation of non-interacting rod-like chains, the effect of the chains on diffusion and magnetophoretic transport in ferrofluids has been studied. Analysis shows that the appearance of the chains leads to strong anisotropy of the diffusion properties of ferrofluids - the effective coefficient of the particle diffusion in the direction along the applied field is about one to two orders of magnitude more than that in the direction perpendicular to the field. This effect is explained by attraction of the particles to the regions where local magnetic field is relatively large, i.e. the concentration of the particles is small.

The appearance of the chains significantly affects the magnetophoretic coefficient of the particles. When the applied magnetic field is small enough, the chains increase this coefficient; when the field is moderate or strong they cause a decrease.

In spite of the fact that the approximation of the non-interacting rod-like chains is a strong simplification of the problem, the same approximation earlier allowed us to describe rheological properties of various ferrofluids. It gives us a background to consider this approximation as a robust foundation for the further study of the transport phenomena in ferrofluids with strongly interacting particles, able to form chains (for example, in fluids with cobalt particles; with cluster particles, etc.). Indeed, analysis [19] shows that this approximation leads to quite reasonable estimates for the magnetic moment of a chain. Although magnetic and sterical interactions between the chains influence the function $g_{n}$ of distribution over 
the number of particles in the chains, this effect is not strong [22]. Thus, in the first approximation, it can be neglected. As for hydrodynamical interactions of the chains, the successful application of the model of non-interacting chains to a theoretical description of the rheological properties of ferrofluids shows that this interaction does not play the principal role in dynamical phenomena in ferrofluids.

One needs to note that when both the concentration and energy of the magnetic interaction of the particles are high enough, they can condense into dense drop-like aggregates. Analysis of the transport phenomena in a system with drops is especially worth studying.

\section{Acknowledgment}

This work has been supported by the grants of the Russian Fund of Fundamental Investigations, NN 10-01-96002-Ural, 10-02-96001-Ural, 10-02-00034.

\section{References}

[1] Yu.A. Buevich, A.Yu. Zubarev, A.O. Ivanov, Magnetohydrodinamics 2 (1989) 39.

[2] K.I. Morozov, J. Magn. Magn. Mater. 122 (1993) 98.

[3] K.I. Morozov, Phys. Rev. E 3 (1996) 3841.

[4] A.F. Pshenichnikov, E.A. Elfımova, A.O. Ivanov, J. Chem. Phys. 134 (2011) 184508

[5] C.F. Hayers, J. Colloid Interface Sci. 52 (1975) 239.

[6] J.C. Bacri, D. Salin, J. Magn. Magn. Matter. 9 (1983) 48.

[7] M.F. Islam, K.H. Lin, D. Lacoste, T.C. Lubenski, A.G. Yodh, Phys. Rev. E 67 (2003) 021402.

[8] M. Klokkenburg, R.P.A. Dullens, W.K. Kegel, B.H. Erne, A.P. Philipse, Phys. Rev. Lett. 96 (2006) 037203.

[9] M. Klokkenburg, B.H. Erne, J.D. Meedldijk, A. Wiedenmann, A.V. Petukhov, R.P.A. Dullens, A.P. Phylipse, Phys. Rev. Lett. 97 (2006) 185702.

[10] S. Odenbach, Magnetoviscous Effects in Ferrofluids, Springer, Berlin, 2002.

[11] P. Ilg, S. Odenbach, in: S. Odenbach (Ed.), Colloidal Magnetic Fluids, Springer, Berlin, 2009.

[12] S. Dutz, W. Andrea, R. Hergt, R. Muller, Ch. Oestreich, Ch. Schmidt, J.T. Eopfer, M. Zeisberger, M. Bellemann, J. Magn. Magn. Mater. 311 (2007) 51.

[13] D. Borin, S. Odenbach, Proceedings of 12th International Conference on Electrorheological Fluids and Magnetorheological Suspensions, World Scientific, 2011

[14] A.Yu. Zubarev, L.Yu. Iskakova, JETP 80 (1995) 857.

[15] A.Yu. Zubarev, J. Fleisher, S. Odenbach, Physica A 358 (2005) 475.

[16] D.N. Chirikov, S.P. Fedotov, L.Yu. Iskakova, A.Yu. Zubarev, Phys. Rev. E 82 (2010) 051405.

[17] D. Borin, A. Zubarev, D. Chirikov, R. Müller, S. Odenbach, J. Magn. Magn. Mater. 323 (2011) 1273.

[18] L. Rodriguez-Arco, M.T. Lopez-Lopez, J.D.G. Duran, A. Zubarev, D. Chirikov, J. Phys.: Condens. Matter 23 (2011) 455101.

[19] V.S. Mendelev, A.O. Ivanov, Phys. Rev. E 70 (2004) 051502.

[20] G.K. Batchelor, J. Fluid Mech. 74 (1976) 1.

[21] G.M. Theo van de Ven, Colloidal Hydrodynamics, Academic Press, 1989.

[22] L.Yu. Iskakova, A.Yu. Zubarev, Phys. Rev. E 66 (2002) 041405. 